Supplement of Magn. Reson., 1, 105-113, 2020

https://doi.org/10.5194/mr-1-105-2020-supplement

(C) Author(s) 2020. This work is distributed under

the Creative Commons Attribution 4.0 License.

(c) (i)

Supplement of

\title{
ArduiTaM: accurate and inexpensive NMR auto tune and match system
}

Mazin Jouda et al.

Correspondence to: Jan G. Korvink (korvink@kit.edu)

The copyright of individual parts of the supplement might differ from the CC BY 4.0 License. 


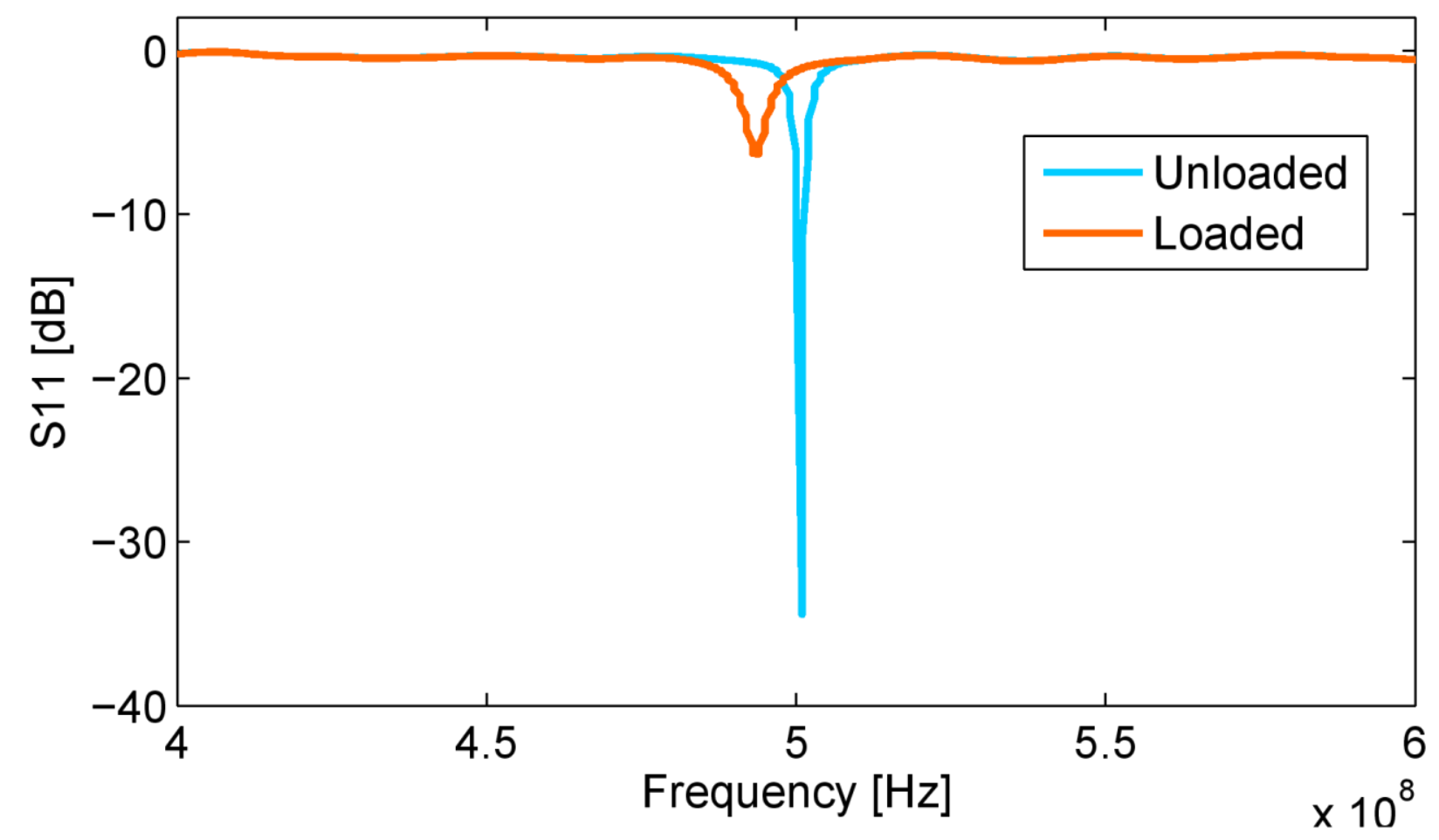

Fig. S1: Effect of sample loading on the tuning and matching conditions. The coil is a commercial $5 \mathrm{~mm}$ saddle coil designed to operate in a $11.7 \mathrm{~T}$ Bruker magnet at $500 \mathrm{MHz}$. Loading the sample (0.5 M NaPO3, 0.5 M Phosphoric Buffer Solution (PBS), $50 \mathrm{mM}$ TSP, 0.5 M Sucrose) disturbs the tuning and matching condition largely (6 MHz frequency shift, $28 \mathrm{~dB}$ change in S11). 


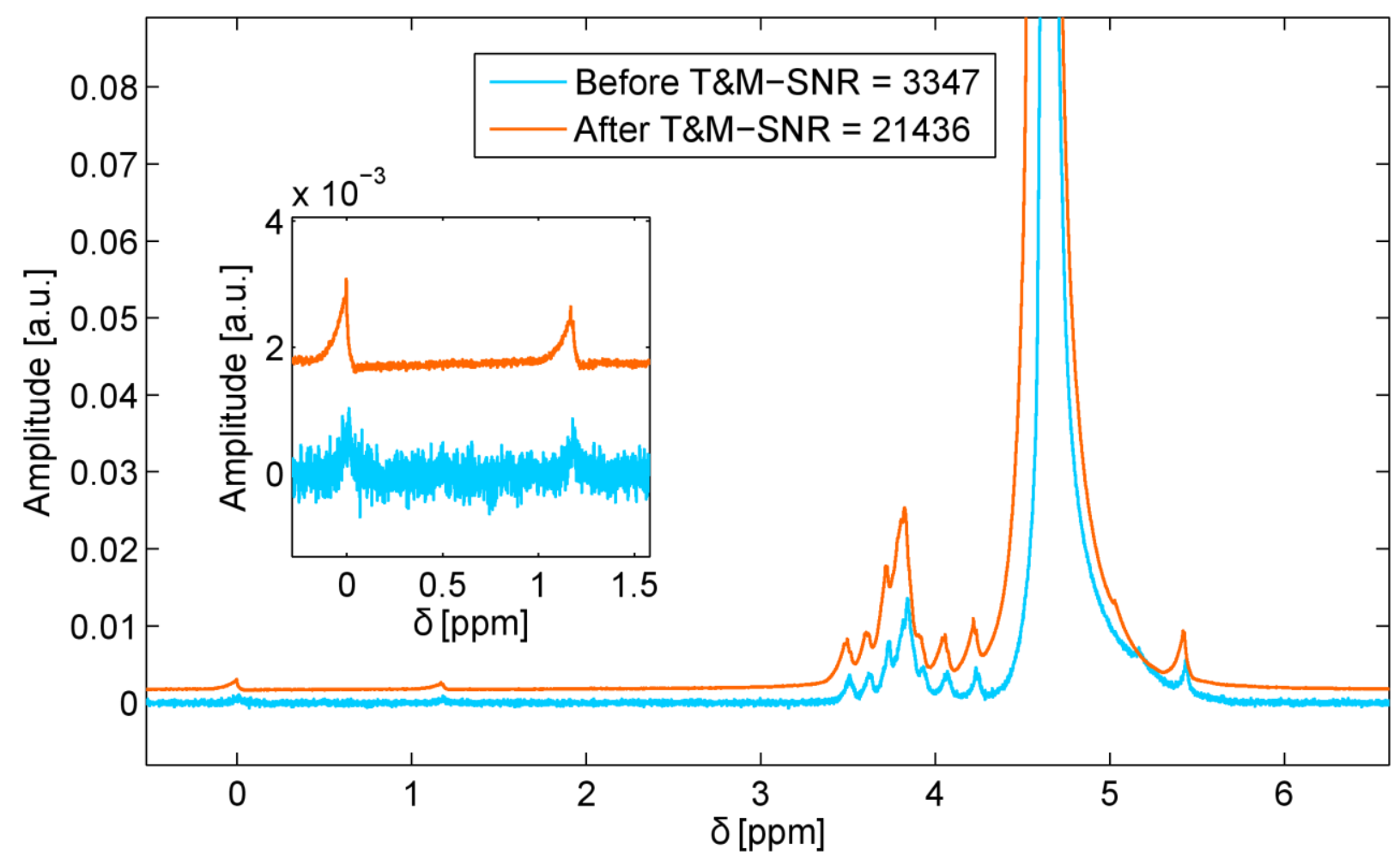

Fig. S2: Effect of tuning and matching on the quality of the NMR spectrum. The coil is a commercial $5 \mathrm{~mm}$ saddle coil designed to operate in a $11.7 \mathrm{~T}$ Bruker magnet at $500 \mathrm{MHz}$. The sample is 0.5 M NaPO3, 0.5 M Phosphoric Buffer Solution (PBS), $50 \mathrm{mM}$ TSP, 0.5 M Sucrose. Readjusting the tuning and matching condition after loading the sample enhanced the SNR significantly (a factor of 6.4). 


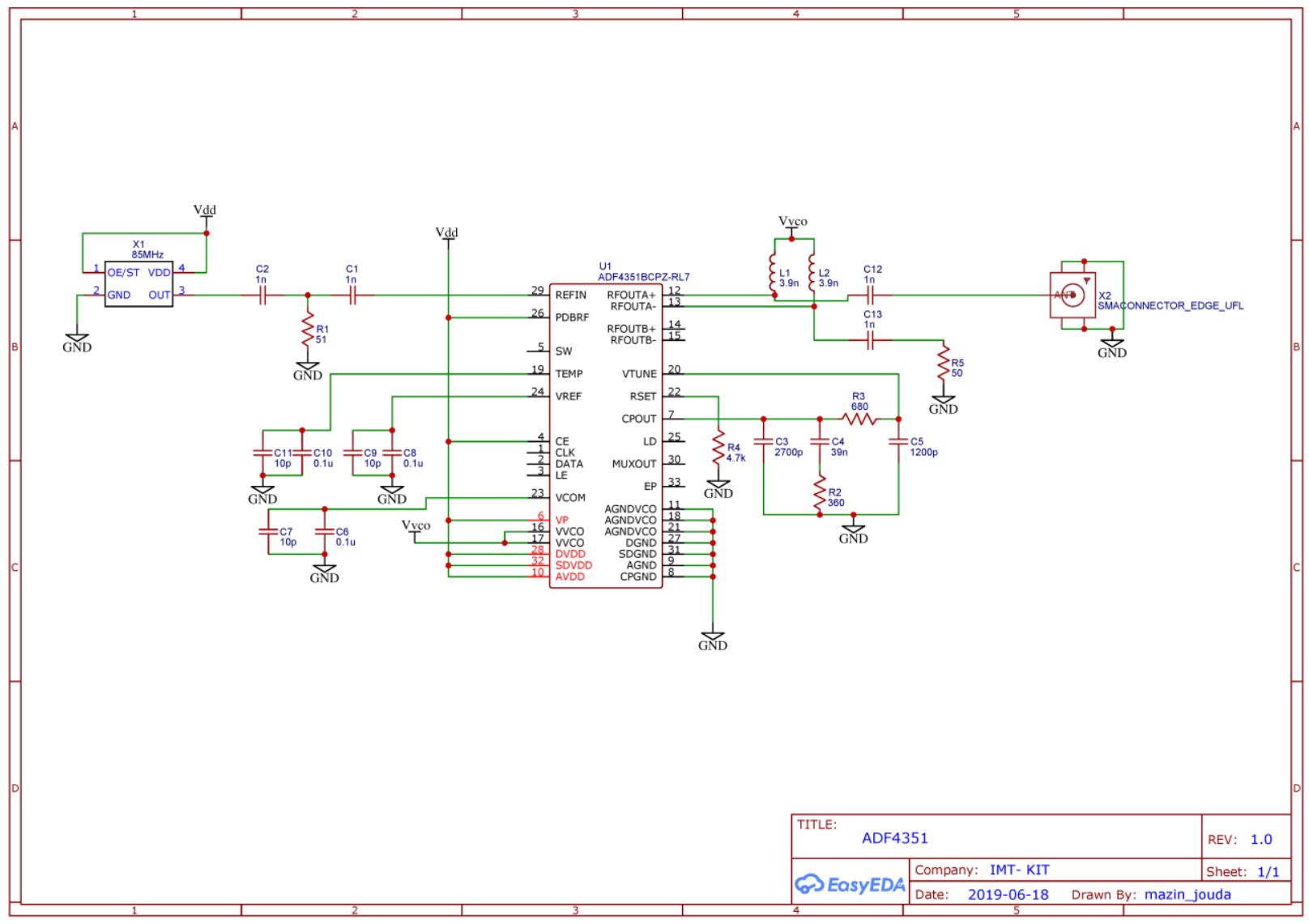

Fig. S3: Schematic diagram of the ADF4351 broadband frequency synthesizer. 


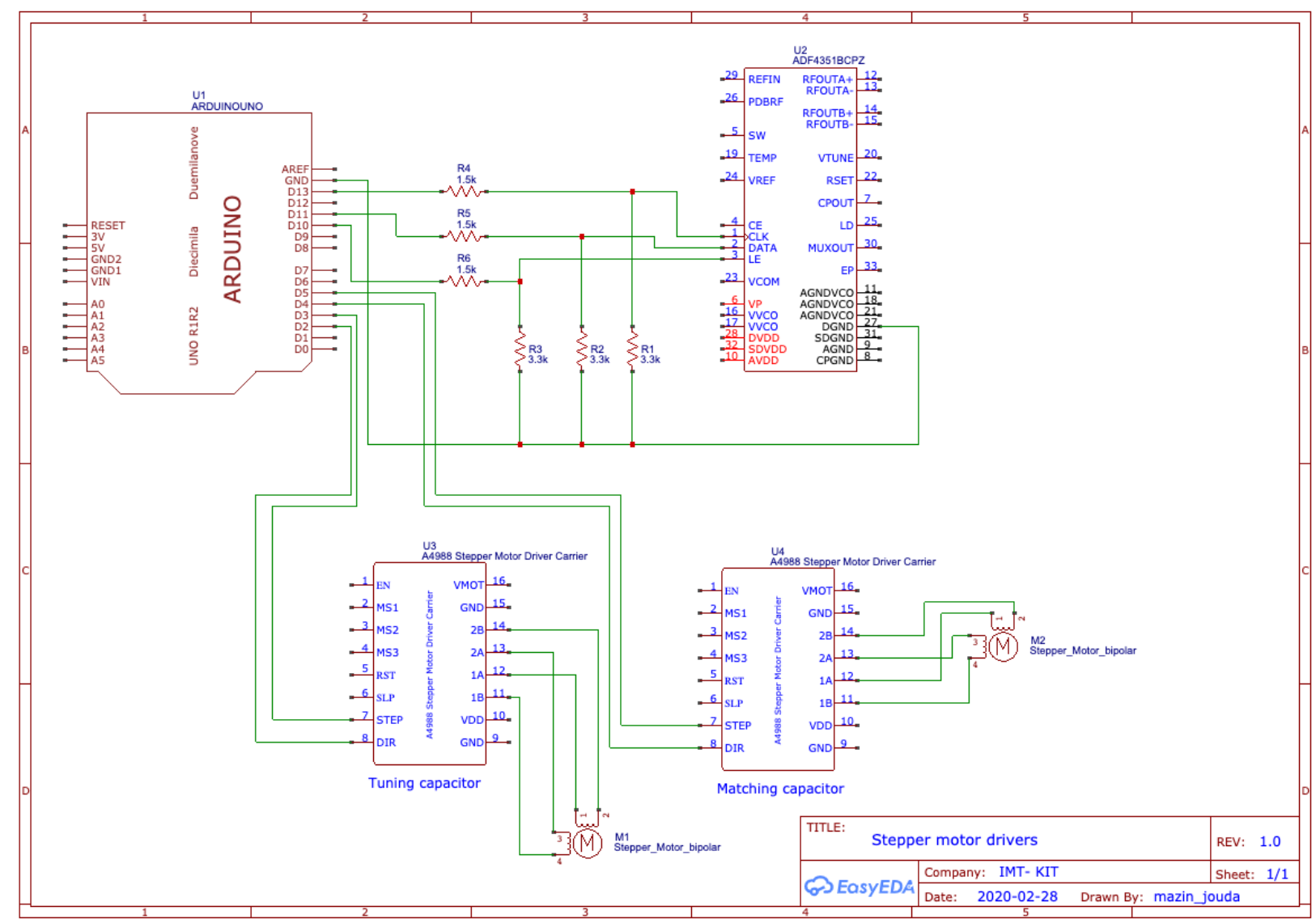

Fig. S4: Circuit diagram describing the interface between the Arduino and the ADF4351 frequency synthesizer. The diagram shows also the connections of the stepper motors' drivers (A4988). 


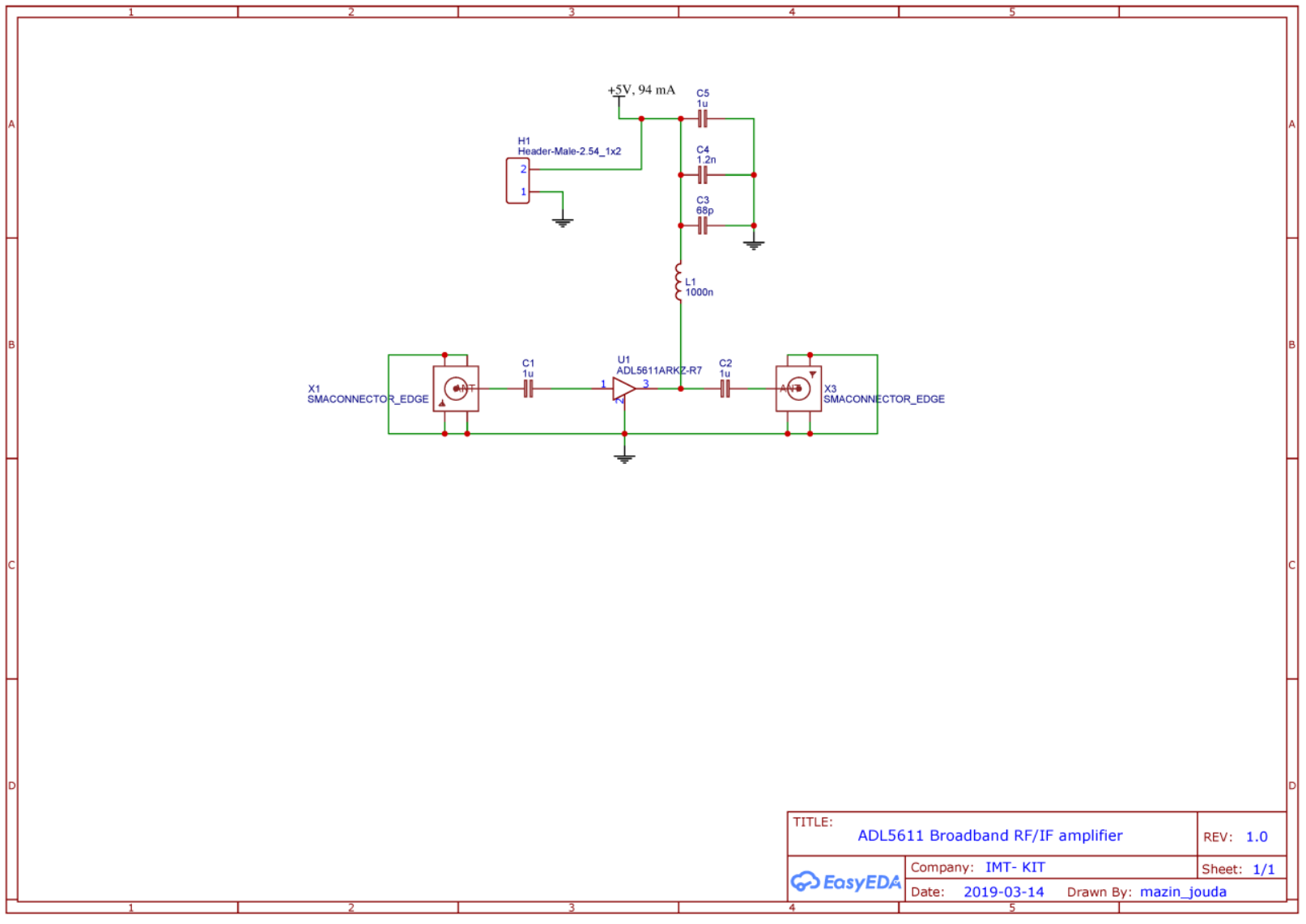

Fig. S5: Circuit diagram of the ADL5611 broadband IF/RF amplifier. 


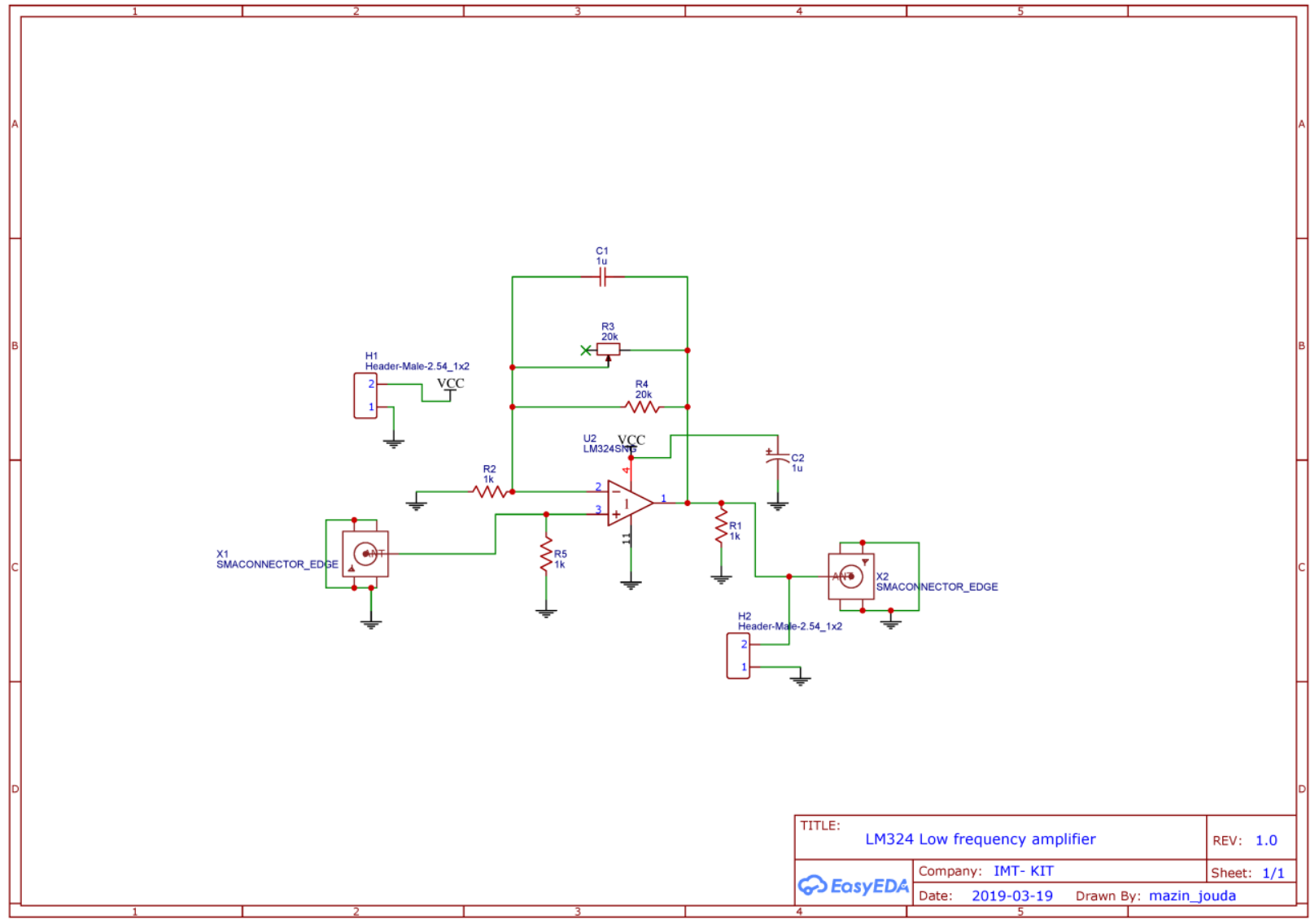

Fig. S6: Schematic of the LM324 low-frequency amplifier. 